\title{
INTEGRAL MEANS FOR THE $n$ 'th DERIVATIVE OF BLASCHKE PRODUCTS
}

\author{
Mirwan Amin Kutbi
}

\section{Preliminaries}

A Blaschke product $B(z)$ associated with the sequence $\left\{a_{k}\right\}$ is a function defined by the formula

$$
B(z)=\prod_{k=1}^{\infty} \frac{\bar{a}_{k}}{\left|a_{k}\right|}\left(\frac{a_{k}-z}{1-\bar{a}_{k} z}\right)
$$

where $\sum_{k=1}^{\infty}\left(1-\left|a_{k}\right|\right)<\infty$, and $0<\left|a_{k}\right|<1$ for all $k$ in $N$. This function is regular and bounded whenever $z$ is in $U=\{z:|z|<1\}$.

For $0<p<\infty$, the Hardy space $H^{p}$ is defined to be the class of all functions $f$ regular in $U$ for which $\sup _{0<r<1}\left\{(1 / 2 \pi) \int_{0}^{2 \pi}\left|f\left(r e^{i \theta}\right)\right|^{p} d \theta\right\}^{1 / p}$ is finite.

In our paper [3], we have shown that if, for some $\alpha$ in $(0,1 / 2)$, we have

$$
\sum_{k=1}^{\infty}\left(1-\left|a_{k}\right|\right)^{\alpha}<\infty
$$

then Protas' consideration [5] of the boundedness of the integral means for the first derivative of Blaschke products $I(r)=\int_{0}^{2 \pi}\left|B^{\prime}\left(r e^{i \theta}\right)\right|^{p} d \theta$, when $0<p \leq 1-\alpha$ can be extended for $p>1-\alpha$.

In 1976 C. N. Linden [4] gave a generalization to higher order derivatives of a theorem of Protas, which gives a sufficient condition for the $n^{\prime}$ th derivative $B^{(n)}(z)$ of an infinite Blaschke product $B(z)$ to belong to the class $H^{p}$ for each $p$ in $(0,(1-\alpha) / n]$ when $\alpha$ is in $(0,1 /(n+1))$. He also gave some relevant counterexamples to indicate to some extent that his results are the best obtainable for general Blaschke products.

In this paper we extend Linden's theorem [4] when $n \in N$ and $p>(1-\alpha) / n$ where $\alpha$ is in $(0,1 /(n+1))$. To do this we extend our results obtained in [3] by considering upper bounds to the integral means of the $n$ 'th derivative of certain infinite Blaschke products for $n>1$.

We note that in $1984 \mathrm{Kim}$ [2] showed that if (2) holds for some $\alpha$ in $(0,1 /(n+1))$, then $B^{(n)}(z) \in H^{p}$ for all $p$ in $(0,(1-\alpha) / n]$. Our results obtained when $p>(1-\alpha) / n$ show that $B^{(n)}(z)$ need not belong to $H^{p}$.

Received April 6, 2001; revised April 1, 2002. 
The positive results are in the form of "little oh" inequalities and, as in the case $n=1$ (see [3]), we indicate the extent to which these can be regarded as being best possible by considering some counterexamples.

Throughout this paper, the symbol $C$ denotes a positive constant, and $C(\cdots)$ a positive constant depending on the parameters indicated in $(\cdots)$. For clarification we will, from time to time, distinguish various appearances of the constants by using suffixes such as $C_{1}, C_{2}, \ldots, C_{1}(\cdots), C_{2}(\cdots), \ldots$ Also the symbol $\varepsilon(r)$ denotes a positive function that tends to zero as $r$ increases to one. However it should be noted that for $j=1,2, \ldots$, the terms $C, C_{j}, C_{j}(\cdots)$ and $\varepsilon(r)$ may vary from one appearance to the next, but always there will be some implicit means of finding them at each stage.

\section{2. n'th derivative means for $(1-\alpha) / n<p \leq 1 / n$}

Here, in the following theorem, we extend Linden's theorem [4] for any natural number $n$ with $(1-\alpha) / n<p \leq 1 / n$ when $\alpha$ is in $(0,1 /(n+1))$ as follows.

THEOREM 1. Let $n$ be a natural number, and let $\left\{a_{k}\right\}$ be a Blaschke sequence such that (2) holds for some $\alpha$ in $(0,1 /(n+1))$. Then, for any number $p$ in $((1-\alpha) / n, 1 / n]$, there is a positive function $\varepsilon(r)$ that satisfies the condition

$$
\lim _{r \rightarrow 1} \varepsilon(r)=0,
$$

and such that

$$
I_{n}(r)=\int_{0}^{2 \pi}\left|B^{(n)}\left(r e^{i \theta}\right)\right|^{p} d \theta<\frac{\varepsilon(r)}{(1-r)^{n p+\alpha-1}} \quad(0<r<1) .
$$

For the proof of this theorem we need the following lemmas. First we have a well-known result.

Lemma 1 (See [6], p. 226). If $x \in(0,1) \cup(1, \infty)$, and $y$ is a natural number, then there are two positive constants $C_{1}(x)$ and $C_{2}(x)$ not dependent on $y$ such that

$$
C_{2}(x)<(1-r)^{h(x)} \int_{0}^{2 \pi}\left|1-r e^{i y \theta}\right|^{-x} d \theta<C_{1}(x) \quad(0<r<1),
$$

where $h(x)=\max (0, x-1)$.

In the next lemma, we generalize our result in Lemma 3 [3], for any natural number $J$, and because of similarity the proof has been omitted.

LEMMA 2. Let $J$ be a natural number, and let $\left\{a_{k}\right\}$ be a Blaschke sequence such that (2) holds for some $\alpha$ in $(0,1)$. Then for any number $p$ satisfying the conditions $p \geq \alpha$ and $J p+\alpha>1$, we have 
INTEGRAL MEANS FOR THE $n$ 'th DERIVATIVE OF BLASCHKE PRODUCTS

$$
\sum_{k=1}^{\infty} \frac{\left(1-r_{k}\right)^{p}}{\left(1-r_{k} r\right)^{(J+1) p-1}}<\frac{\varepsilon(r)}{(1-r)^{J p+\alpha-1}} \quad(0<r<1),
$$

where $r_{k}=\left|a_{k}\right|$ and $\lim _{r \rightarrow 1} \varepsilon(r)=0$.

In the following lemma, we establish the general derivative formula for the product $B(z)$.

Lemma 3. Let $B(z)$ be a Blaschke product. Then, for $J=0,1, \ldots$, we have

$$
B^{(J+1)}(z)=-\sum_{s=0}^{J} \sum_{k=1}^{\infty} \frac{J !(s+1)}{(J-s) !} B_{k}^{(J-s)}(z) \frac{\left(\bar{a}_{k}\right)^{s+1}\left(1-\left|a_{k}\right|^{2}\right)}{\left|a_{k}\right|\left(1-\bar{a}_{k} z\right)^{s+2}},
$$

where

$$
B_{k}(z)=\prod_{\substack{j=1 \\ j \neq k}}^{\infty} \frac{\bar{a}_{j}}{\left|a_{j}\right|}\left(\frac{a_{j}-z}{1-\bar{a}_{j} z}\right) .
$$

Proof. The logarithmic derivative of $B(z)$ can be used to give the formula

$$
B^{\prime}(z)=-\sum_{k=1}^{\infty} B_{k}(z) D_{k}(z)
$$

where

$$
D_{k}(z)=\left(\frac{\bar{a}_{k}}{\left|a_{k}\right|}\right) \frac{1-\left|a_{k}\right|^{2}}{\left(1-\bar{a}_{k} z\right)^{2}} .
$$

Then Leibnitz's formula [1, p. 121] shows that

$$
\begin{aligned}
B^{(J+1)}(z) & =\frac{d^{J}}{d z^{J}} B^{\prime}(z)=-\sum_{k=1}^{\infty} \frac{d^{J}}{d z^{J}}\left\{B_{k}(z) D_{k}(z)\right\} \\
& =-\sum_{k=1}^{\infty}\left\{\sum_{s=0}^{J} \frac{J !}{s !(J-s) !} B_{k}^{(J-s)}(z) D_{k}^{(s)}(z)\right\} .
\end{aligned}
$$

Note that, a simple application of the principle of mathematical induction can be used to establish the formula

$$
D_{k}^{(s)}(z)=\frac{(s+1) !\left(\bar{a}_{k}\right)^{s+1}\left(1-\left|a_{k}\right|^{2}\right)}{\left|a_{k}\right|\left(1-\bar{a}_{k} z\right)^{s+2}} \quad(s=0,1,2, \ldots) .
$$

Substituting (10) in (9) completes the proof.

The next two lemmas give upper bounds to the quantities $\left|B^{(J)}(z)\right|$ and $I_{J}(r)=\int_{0}^{2 \pi}\left|B^{(J)}\left(r e^{i \theta}\right)\right|^{p} d \theta(1 /(J+1)<p \leq 1 / J, J \in \boldsymbol{N})$, respectively. These re- 
sults are actually due to Kim [2] but we give an alternative inductive proof which does not require the more complicated appeal to partial products used by him.

Lemma 4. Let $B(z)$ be a Blaschke product. Then, for any natural number $J$, we have

$$
\left|B^{(J)}(z)\right|<C(J) \sum^{*}\left[\prod_{s=1}^{l}\left\{\sum_{k=1}^{\infty} \frac{1-\left|a_{k}\right|}{\left|1-\bar{a}_{k} z\right|^{1+\lambda_{s}}}\right\}\right],
$$

where $\sum^{*}$ is a finite sum taken over all l-tuples $\left(\lambda_{1}, \ldots, \lambda_{s}, \ldots, \lambda_{l}\right)(l \leq J)$ for which

$$
\lambda_{1}+\cdots+\lambda_{s}+\cdots+\lambda_{l}=J
$$

and

$$
1 \leq \lambda_{s} \leq J \text { for } 1 \leq s \leq l .
$$

Proof. We proceed by using the principle of mathematical induction. The required result is true for $J=1$, since

$$
\left|B^{\prime}(z)\right|<2 \sum_{k=1}^{\infty} \frac{1-\left|a_{k}\right|}{\left|1-\bar{a}_{k} z\right|^{2}}
$$

(see Theorem 1 [3]).

Suppose that the conclusion of Lemma 4 is valid for all positive integers up to $J$. Then by Lemma 3 we obtain

$$
\begin{aligned}
\left|B^{(J+1)}(z)\right| & =\left|-\sum_{h=0}^{J} \sum_{k=1}^{\infty} \frac{J !(h+1)}{(J-h) !} \frac{\left(\bar{a}_{k}\right)^{h+1}\left(1-\left|a_{k}\right|^{2}\right)}{\left|a_{k}\right|\left(1-\bar{a}_{k} z\right)^{h+2}} B_{k}^{(J-h)}(z)\right| \\
& <T_{1}(z)+T_{2}(z),
\end{aligned}
$$

where

$$
\begin{aligned}
T_{1}(z)= & 2 \sum_{h=0}^{J-1} \frac{J !(h+1)}{(J-h) !} \sum_{k=1}^{\infty} \frac{1-\left|a_{k}\right|}{\left|1-\bar{a}_{k} z\right|^{h+2}}\left|B_{k}^{(J-h)}(z)\right| \\
< & 2 \sum_{h=0}^{J-1} \frac{J !(h+1)}{(J-h) !} \sum_{k=1}^{\infty} \frac{1-\left|a_{k}\right|}{\left|1-\bar{a}_{k} z\right|^{1+(h+1)}} \\
& \times C(J-h) \sum^{* *}\left[\prod_{s=1}^{l}\left\{\sum_{k=1}^{\infty} \frac{1-\left|a_{k}\right|}{\left|1-\bar{a}_{k} z\right|^{1+\lambda_{s}}}\right\}\right] \\
< & C(J) \sum_{*}\left[\prod_{s=1}^{L}\left\{\sum_{k=1}^{\infty} \frac{1-\left|a_{k}\right|}{\left|1-\bar{a}_{k} z\right|^{1+\lambda_{s}}}\right\}\right],
\end{aligned}
$$


INTEGRAL MEANS FOR THE $n$ 'th DERIVATIVE OF BLASCHKE PRODUCTS

the sum $\sum^{* *}$ in $(14)$ being taken over all $l$-tuples $\left(\lambda_{1}, \ldots, \lambda_{s}, \ldots, \lambda_{l}\right)$ such that, for given $h$ in $[0, J-1], \lambda_{1}+\cdots+\lambda_{s}+\cdots+\lambda_{l}=J-h$ and $1 \leq \lambda_{s} \leq J-h$ for $1 \leq s \leq l \leq J-h$, while the sum $\sum_{*}$ in (15) is taken over all $L$-tuples $\left(\lambda_{1}, \ldots, \lambda_{s}, \ldots, \lambda_{L}\right)$ for which $\lambda_{1}+\cdots+\lambda_{s}^{*}+\cdots+\lambda_{L}=J+1$ and $1 \leq \lambda_{s} \leq J+1$ for $1 \leq s \leq L \leq J+1$.

Next, we consider

$$
\begin{aligned}
T_{2}(z) & =2\{(J+1) !\} \sum_{k=1}^{\infty} \frac{1-\left|a_{k}\right|}{\left|1-\bar{a}_{k} z\right|^{J+2}}\left|B_{k}(z)\right| \\
& <2\{(J+1) !\} \sum_{k=1}^{\infty} \frac{1-\left|a_{k}\right|}{\left|1-\bar{a}_{k} z\right|^{1+(J+1)}},
\end{aligned}
$$

since $\left|B_{k}(z)\right|<1$.

Thus, substituting (15) and (16) in (13) and replacing the constants $C(J)$ and $2\{(J+1) !\}$ by a bigger constant $C(J+1)$ end the proof of Lemma 4 .

Lemma 5. Let $J$ be a natural number, and let $1 /(J+1)<p \leq 1 / J$. Then

$$
I_{J}(r)=\int_{0}^{2 \pi}\left|B^{(J)}\left(r e^{i \theta}\right)\right|^{p} d \theta<C(J, p) \sum_{k=1}^{\infty} \frac{\left(1-r_{k}\right)^{p}}{\left(1-r_{k} r\right)^{(J+1) p-1}},
$$

where $a_{k}=r_{k} e^{i \gamma_{k}}$.

Proof. Since $p \leq 1$, then by Lemma 4 we get

$$
\left|B^{(J)}(z)\right|^{p}<C(J, p) \sum^{*}\left[\prod_{s=1}^{l}\left\{\sum_{k=1}^{\infty} \frac{1-\left|a_{k}\right|}{\left|1-\bar{a}_{k} z\right|^{1+\lambda_{s}}}\right\}^{p}\right] .
$$

Now we need to examine terms of the form

$$
T(z)=\prod_{s=1}^{l}\left\{\sum_{k=1}^{\infty} \frac{1-\left|a_{k}\right|}{\left|1-\bar{a}_{k} z\right|^{1+\lambda_{s}}}\right\}^{p} .
$$

We apply Hölder's inequality to get

$$
\int_{0}^{2 \pi} T\left(r e^{i \theta}\right) d \theta \leq \prod_{s=1}^{l}\left[\int_{0}^{2 \pi}\left\{\sum_{k=1}^{\infty} \frac{1-r_{k}}{\left|1-r_{k} r e^{i\left(\theta-\gamma_{k}\right)}\right|^{1+\lambda_{s}}}\right\}^{t_{s} p} d \theta\right]^{1 / t_{s}},
$$

where $t_{s}=J / \lambda_{s}$.

Since $t_{s} p=J p / \lambda_{s} \leq J p \leq 1$, then for $\omega=\theta-\gamma_{k}$ we get

$$
\int_{0}^{2 \pi}\left\{\sum_{k=1}^{\infty} \frac{1-r_{k}}{\left|1-r_{k} r e^{i\left(\theta-\gamma_{k}\right)}\right|^{1+\lambda_{s}}}\right\}^{t_{s} p} d \theta \leq \sum_{k=1}^{\infty} \int_{0}^{2 \pi} \frac{\left(1-r_{k}\right)^{t_{s} p}}{\left|1-r_{k} r e^{i \omega}\right|^{\left(1+\lambda_{s}\right) t_{s} p}} d \omega .
$$

The inequalities $1 \leq \lambda_{s} \leq J$ and $p>1 /(J+1)$ imply 


$$
\left(1+\lambda_{s}\right) t_{s} p=\left(J+\frac{J}{\lambda_{s}}\right) p \geq(J+1) p>1 .
$$

Therefore, Lemma 1 yields

$$
\int_{0}^{2 \pi}\left|1-r_{k} r e^{i \omega}\right|^{-\left(1+\lambda_{s}\right) t_{s} p} d \omega<C(J, p)\left(1-r_{k} r\right)^{1-\left(1+\lambda_{s}\right) t_{s} p},
$$

and since $\left(\left(1-r_{k}\right) /\left(1-r_{k} r\right)\right)^{p\left(\left(J-\lambda_{s}\right) / \lambda_{s}\right)} \leq 1$ for all $J \geq \lambda_{s}$, it follows that

$$
\begin{aligned}
& \sum_{k=1}^{\infty} \int_{0}^{2 \pi} \frac{\left(1-r_{k}\right)^{t_{s} p}}{\left|1-r_{k} r e^{i \omega \mid}\right|^{\left(1+\lambda_{s}\right) t_{s} p}} d \omega<C(J, p) \sum_{k=1}^{\infty} \frac{\left(1-r_{k}\right)^{J p / \lambda_{s}}}{\left(1-r_{k} r\right)^{\left(\left(1+\lambda_{s}\right) J p\right) / \lambda_{s}-1}} \\
& \quad=C(J, p) \sum_{k=1}^{\infty} \frac{\left(1-r_{k}\right)^{p}}{\left(1-r_{k} r\right)^{(J+1) p-1}}\left(\frac{1-r_{k}}{1-r_{k} r}\right)^{p\left(\left(J-\lambda_{s}\right) / \lambda_{s}\right)} \\
& \quad \leq C(J, p) \sum_{k=1}^{\infty} \frac{\left(1-r_{k}\right)^{p}}{\left(1-r_{k} r\right)^{(J+1) p-1}} .
\end{aligned}
$$

Note that,

$$
\sum_{s=1}^{l} \frac{1}{t_{s}}=\frac{1}{J} \sum_{s=1}^{l} \lambda_{s}=1 .
$$

Therefore, (20), (21) and (23) imply

$$
\begin{aligned}
\int_{0}^{2 \pi} T\left(r e^{i \theta}\right) d \theta & <\prod_{s=1}^{l}\left\{C(J, p) \sum_{k=1}^{\infty} \frac{\left(1-r_{k}\right)^{p}}{\left(1-r_{k} r\right)^{(J+1) p-1}}\right\}^{1 / t_{s}} \\
& <C(J, p) \sum_{k=1}^{\infty} \frac{\left(1-r_{k}\right)^{p}}{\left(1-r_{k} r\right)^{(J+1) p-1}} .
\end{aligned}
$$

Together with (18), this completes the proof of Lemma 5.

The proof of Theorem 1 follows since the result (4) is implied by using, respectively, Lemma 5 and Lemma 2 with $n$ in place of $J$, and noting that $p>\alpha$ with $J p+\alpha-1>0$.

\section{3. $n$ 'th derivative means for $p>1 / n$}

In the following theorem we extend Theorem 1 when $0<\alpha<1 /(n+1)$ $(n \in \boldsymbol{N})$ and $p>1 / n$ as follows.

THEOREM 2. Let $n$ be a natural number, and let $\left\{a_{k}\right\}$ be a Blaschke sequence such that (2) holds for some $\alpha$ in $(0,1 /(n+1))$. Then, for any number $p>1 / n$, there is a positive function $\varepsilon(r)$ that satisfies the condition (3) and such that 
INTEGRAL MEANS FOR THE $n$ 'th DERIVATIVE OF BLASCHKE PRODUCTS

$$
I_{n}(r)=\int_{0}^{2 \pi}\left|B^{(n)}\left(r e^{i \theta}\right)\right|^{p} d \theta<\frac{\varepsilon(r)}{(1-r)^{n p+\alpha-1}} \quad(0<r<1) .
$$

Let us first state the following lemma.

Lemma 6. Let $B(z)$ be a Blaschke product. Then, for any $z \in U=$ $\{z:|z|<1\}$

$$
\left|B^{(n)}(z)\right|<\frac{C(n)}{(1-r)^{n}} \quad(|z|=r)
$$

(see Lemma 4 [3] for the case $n=1$ ).

We are now ready to prove Theorem 2. For a suitable choice of a number $\gamma$ so that $(1-\alpha) / n<\gamma \leq 1 / n$, Lemma 6 together with Theorem 1 imply

$$
I_{n}(r)=\int_{0}^{2 \pi}\left|B^{(n)}\left(r e^{i \theta}\right)\right|^{p} d \theta<\frac{C(n, \gamma, p)}{(1-r)^{n(p-\gamma)}} \frac{\varepsilon(r)}{(1-r)^{n \gamma+\alpha-1}} \leq \frac{\varepsilon(r)}{(1-r)^{n p+\alpha-1}},
$$

if $C(n, \gamma, p) \leq 1$. If not, then replacing $\varepsilon(r)$ by $\varepsilon(r) / C(n, \gamma, p)$ initially completes the proof.

\section{General counterexamples in cases when $(1-a) / n<p \leq 1 / n$}

Next theorem indicates the extent to which the inequality (4) with the condition (3) is the best possible result that can be obtained for general infinite Blaschke products.

THEOREM 3. For a natural number $n$ let $\alpha$ be in $(0,1 /(n+1))$, and let $\varepsilon(r)$ be a given positive function which is continuous on $(0,1)$ and satisfies the condition (3). Then we can find an infinite Blaschke product $B(z)$ whose zeros $\left\{a_{k}\right\}$ satisfy the associated convergence condition (2), and for any number $p$ in $((1-\alpha) / n, 1 / n]$ we have

$$
I_{n}(r)=\int_{0}^{2 \pi}\left|B^{(n)}\left(r e^{i \theta}\right)\right|^{p} d \theta>\frac{\varepsilon(r)}{(1-r)^{n p+\alpha-1}},
$$

for a sequence of values $r$ increasing to 1 .

To prove this theorem, we may consider a sequence of numbers $\left\{r_{k}\right\}$ increasing to 1 on $(0,1)$, and an associated sequence of integers $\left\{q_{k}\right\}$ so that the Blaschke product $B(z)$ has $q_{k}$ zeros of the form $r_{k} e^{i 2 \pi j / q_{k}}\left(0 \leq j \leq q_{k}-1\right)$ equally distributed on $\left\{z:|z|=r_{k}\right\}$, according to the following definition:

$$
B(z)=\prod_{k=1}^{\infty} \frac{r_{k}^{q_{k}}-z^{q_{k}}}{1-r_{k}^{q_{k}} z^{q_{k}}}=\prod_{k=1}^{\infty} b_{k}(z)
$$


We will make further qualifications on these sequences later. Note, however, that the general convergence condition (2) will be equivalent to

$$
\sum_{k=1}^{\infty} q_{k}\left(1-r_{k}\right)^{\alpha}<\infty
$$

for some $\alpha$ in $(0,1 /(n+1))$ and $n \in \boldsymbol{N}$, and we will assume that this is so.

For a number $\sigma$ in $(0,1)$, we will first choose the sequence $\left\{r_{k}\right\}$ in $(0,1)$ so that

$$
1-r_{k+1}=\sigma\left(1-r_{k}\right) \quad(k=1,2, \ldots) .
$$

We continue by assuming, without loss of generality, that the given function $\varepsilon(r)$ is decreasing. If not, then we can replace it by $\varepsilon_{1}(r)=\sup \{\varepsilon(x): x \geq r\}$ which satisfies the same conditions as $\varepsilon(r)$ with the addition that $\varepsilon_{1}(r)$ is decreasing and $\varepsilon_{1}(r) \geq \varepsilon(r)$.

First we state the following lemma which will be used to show that, without loss of generality, we can replace the decreasing function $\varepsilon$ by a smoother function to make our deductions easier to pick out dominant terms in $B(z)$. We omit the proof for brevity.

Lemma 7. For a given positive decreasing function $\varepsilon(r)$ which is continuous on $(0,1)$ with $\lim _{r \rightarrow 1} \varepsilon(r)=0$, there is a positive decreasing function $\delta(r)$ satisfying the conditions:

(i) $\delta(r) \geq \varepsilon(r)(r \in(0,1))$,

(ii) $\lim _{r \rightarrow 1} \delta(r)=0$,

(iii) for each given number $\sigma$ in $(0,1)$

$$
\lim _{r \rightarrow 1} \frac{\delta(r)}{\delta(1-\sigma(1-r))}=1 \text {. }
$$

It would be interesting to note that, under the given conditions, the function $\delta(r)$ cannot be smaller than any positive power of $(1-r)$.

By applying Lemma 7 to $\varepsilon(r)$, we can find $\delta(r)$ which satisfies the conditions (i), (ii), (iii). It will be convenient to prove our result in terms of $\delta$ rather than $\varepsilon$, which will be acceptable in view of condition (i). Consequently we define the associated sequence of integers $\left\{q_{k}\right\}$ so that

$$
q_{k}=\left[\left(1-r_{k}\right)^{-\alpha} \delta\left(r_{k}\right)\right] \quad(k=1,2, \ldots) .
$$

This means that, for large value of $k$, we can write

$$
\frac{\delta\left(r_{k}\right)}{2}<q_{k}\left(1-r_{k}\right)^{\alpha} \leq \delta\left(r_{k}\right) \quad\left(k \geq k_{0} ; k, k_{0} \in N\right) .
$$

Henceforth, we should bear in mind that, our results will be valid as long as $\sum_{k=1}^{\infty} \delta\left(r_{k}\right)$ converges, this, in fact, being equivalent to the convergence of 
$\sum_{k=1}^{\infty} q_{k}\left(1-r_{k}\right)^{\alpha}$. However, our specification of the smooth function $\delta$ does not ensure that this is so. In order to proceed further with such cases, it worth noting that, our obtained results will be hold not only for the sequences $\left\{r_{k}\right\}$ and $\left\{q_{k}\right\}$, but for all their subsequences. We also note that, due to the condition $\lim _{r \rightarrow 1} \delta(r)=0$, we can choose a subsequence of natural numbers $\left\{k_{s}\right\}$ such that

$$
\sum_{s=1}^{\infty} \delta\left(r_{k_{s}}\right)<\infty
$$

This, together with the condition $\delta\left(r_{k_{s}}\right) \geq q_{k_{s}}\left(1-r_{k_{s}}\right)^{\alpha}$ implies that

$$
\sum_{s=1}^{\infty} q_{k_{s}}\left(1-r_{k_{s}}\right)^{\alpha} \leq \sum_{s=1}^{\infty} \delta\left(r_{k_{s}}\right)<\infty .
$$

Further, we note that, the general inequalities obtained relating to $B(z)$ in (26) are also applicable correspondingly to the following Blaschke subproduct:

$$
\beta(z)=\prod_{s=1}^{\infty} \frac{r_{k_{s}}^{q_{k_{s}}}-z^{q_{k_{s}}}}{1-r_{k_{s}}^{q_{k_{s}}} z^{q_{k_{s}}}} .
$$

We proceed by proving the following lemmas. In the first one, which can be easily proved by using the principle of mathematical induction, we establish a general derivative formula for $b_{t}(z)$.

Lemma 8. Let $b_{t}(z)$ be defined as in (26) with $t$ in place of $k$. Then

$$
b_{t}^{(J)}(z)=\frac{-q_{t}\left(r_{t}^{q_{t}}-r_{t}^{-q_{t}}\right)}{z^{J}} \sum_{h=1}^{J+1} \frac{\mu_{h, J}}{\left(1-r_{t}^{q_{t}} z^{q_{t}}\right)^{h}} \quad(J=1,2, \ldots),
$$

where each of $\mu_{h, J}$ is a polynomial of degree at most $(J-1)$ in $q_{t}$ with leading coefficient depending only on $J$, and $\mu_{J+1, J}=J ! q_{t}^{J-1}$.

The next lemma estimates the magnitude of what will be shown to be the dominant term in the contribution of the integral $I_{n}(r)$ in (25) for $r=r_{t}(t \in N)$, when $t$ is large.

Lemma 9. For a natural number $J$ let $p>1 /(J+1)$. Then, for a sequence of values $r=r_{t}\left(t \geq t_{0} ; t, t_{0} \in \boldsymbol{N}\right)$ increasing to 1 , we have

$$
C_{1}(J, p) q_{t}\left(1-r_{t}\right)^{1-J p}<\int_{0}^{2 \pi}\left|b_{t}^{(J)}\left(r_{t} e^{i \theta}\right)\right|^{p} d \theta<C_{2}(J, p) q_{t}\left(1-r_{t}\right)^{1-J p},
$$

where $b_{t}\left(r_{t} e^{i \theta}\right)$ is defined as in (26).

(Note that, for some $\alpha$ in $(0,1)$ we have from (29) that, $q_{t} \leq\left(1-r_{t}\right)^{-\alpha} \delta\left(r_{t}\right)$ with $\left.\lim _{t \rightarrow \infty} \delta\left(r_{t}\right)=0\right)$. 
Proof. Suppose first that $1 /(J+1)<p \leq 1$. By Lemma 8 we get

$$
\int_{0}^{2 \pi}\left|b_{t}^{(J)}\left(r_{t} e^{i \theta}\right)\right|^{p} d \theta \geq \frac{q_{t}^{p}\left(r_{t}^{-q_{t}}-r_{t}^{q_{t}}\right)^{p}}{r_{t}^{J p}}\left\{\begin{array}{l}
\int_{0}^{2 \pi}\left|\frac{\mu_{J+1, J}}{\left(1-r_{t}^{2 q_{t}} e^{i q_{t} \theta}\right)^{J+1}}\right|^{p} d \theta \\
-\sum_{h=1}^{J} \int_{0}^{2 \pi}\left|\frac{\mu_{h, J}}{\left(1-r_{t}^{2 q_{t}} e^{i q_{t} \theta}\right)^{h}}\right|^{p} d \theta
\end{array}\right\} .
$$

Let $F_{1}\left(r_{t}\right)$ and $F_{2}\left(r_{t}\right)$ denote respectively the two terms that make up the righthand bracket of $(33)$. Since $(J+1) p>1$, then Lemma 1 yields

$$
F_{1}\left(r_{t}\right)=\int_{0}^{2 \pi}\left|\frac{\mu_{J+1, J}}{\left(1-r_{t}^{2 q_{t}} e^{i q_{t} \theta}\right)^{J+1}}\right|^{p} d \theta>\frac{C(J, p) q_{t}^{(J-1) p}}{\left(1-r_{t}^{2 q_{t}}\right)^{(J+1) p-1}} .
$$

To estimate $F_{2}\left(r_{t}\right)$ note that, since $\left|1-r_{t}^{2 q_{t}} e^{i q_{t} \theta}\right|<2$, we get

$$
F_{2}\left(r_{t}\right)=\sum_{h=1}^{J} \int_{0}^{2 \pi}\left|\frac{\mu_{h, J}}{\left(1-r_{t}^{2 q_{t}} e^{i q_{t} \theta}\right)^{h}}\right|^{p} d \theta<C_{3}(J, p) q_{t}^{(J-1) p} \int_{0}^{2 \pi}\left|1-r_{t}^{2 q_{t}} e^{i q_{t} \theta}\right|^{-J p} d \theta .
$$

If $J p<1$ then $\int_{0}^{2 \pi}\left|1-r_{t}^{2 q_{t}} e^{i q_{t} \theta}\right|^{-J p} d \theta$ is bounded above by a positive constant. Otherwise we have $1 \leq J p<(J+1 / 2) p$, and it follows that

$$
\int_{0}^{2 \pi}\left|1-r_{t}^{2 q_{t}} e^{i q_{t} \theta}\right|^{-J p} d \theta<C_{4}(J, p)\left(1-r_{t}^{2 q_{t}}\right)^{1-(J+1 / 2) p} .
$$

Thus we obtain

$$
F_{2}\left(r_{t}\right)<C_{5}(J, p) q_{t}^{(J-1) p}\left(1-r_{t}^{2 q_{t}}\right)^{1-(J+1 / 2) p} .
$$

Since $1-r_{t}^{2 q_{t}} \leq 2 q_{t}\left(1-r_{t}\right) \leq 2\left(1-r_{t}\right)^{1-\alpha} \delta\left(r_{t}\right)$, then we get from (34) and (35)

$$
F_{1}\left(r_{t}\right)-F_{2}\left(r_{t}\right)>\frac{q_{t}^{(J-1) p}}{\left(1-r_{t}^{2 q_{t}}\right)^{(J+1) p-1}}\left\{C(J, p)-C_{5}(J, p) 2^{p / 2}\left(1-r_{t}\right)^{(1-\alpha) p / 2}\left(\delta\left(r_{t}\right)\right)^{p / 2}\right\} .
$$

For large numbers $t$, the right-hand side of the last inequality is asymptotically equal to $C(J, p) q_{t}^{(J-1) p} /\left(1-r_{t}^{2 q_{t}}\right)^{(J+1) p-1}$, and substitution in (33) yields

$$
\begin{aligned}
\int_{0}^{2 \pi}\left|b_{t}^{(J)}\left(r_{t} e^{i \theta}\right)\right|^{p} d \theta & >\frac{q_{t}^{p}\left(r_{t}^{-q_{t}}-r_{t}^{q_{t}}\right)^{p}}{r_{t}^{J p}} \frac{C(J, p) q_{t}^{(J-1) p}}{\left(1-r_{t}^{2 q_{t}}\right)^{(J+1) p-1}} \\
& =\frac{q_{t}^{p}\left(1-r_{t}^{2 q_{t}}\right)^{p}}{r_{t}^{\left(q_{t}+J\right) p}} \frac{C(J, p) q_{t}^{(J-1) p}}{\left(1-r_{t}^{2 q_{t}}\right)^{(J+1) p-1}} \\
& \geq C(J, p) 2^{1-J p} r_{t}^{\left(-q_{t}-J\right) p} q_{t}\left(1-r_{t}\right)^{1-J p} \\
& >C_{1}(J, p) q_{t}\left(1-r_{t}\right)^{1-J p} .
\end{aligned}
$$


On the other hand, for $1 /(J+1)<p \leq 1$, and for large numbers $t$, we have

$$
\begin{gathered}
\int_{0}^{2 \pi}\left|b_{t}^{(J)}\left(r_{t} e^{i \theta}\right)\right|^{p} d \theta \leq \frac{q_{t}^{p}\left(r_{t}^{-q_{t}}-r_{t}^{q_{t}}\right)^{p}}{r_{t}^{J p}}\left\{\sum_{h=1}^{J+1} \int_{0}^{2 \pi}\left|\frac{\mu_{h, J}}{\left(1-r_{t}^{2 q_{t}} e^{i q_{t} \theta}\right)^{h}}\right|^{p} d \theta\right\} \\
\leq \frac{q_{t}^{p}\left(r_{t}^{-q_{t}}-r_{t}^{q_{t}}\right)^{p}}{r_{t}^{J p}} C_{6}(J, p) q_{t}^{(J-1) p} \sum_{h=1}^{J+1} \int_{0}^{2 \pi}\left|1-r_{t}^{2 q_{t}} e^{i q_{t} \theta}\right|^{-h p} d \theta \\
<C_{2}(J, p) q_{t}\left(1-r_{t}\right)^{1-J p},
\end{gathered}
$$

since $\left|1-r_{t}^{2 q_{t}} e^{i q_{t} \theta}\right|<2,(J+1) p>1$, and $r_{t}^{q_{t}} \rightarrow 1$ as $t \rightarrow \infty$.

This together with (36), ends the proof of Lemma 9 when $1 /(J+1)<p \leq 1$.

We now consider the case when $p>1$. Hölder's inequality with (34) and (35) yields

$$
\begin{gathered}
\left\{\int_{0}^{2 \pi}\left|b_{t}^{(J)}\left(r_{t} e^{i \theta}\right)\right|^{p} d \theta\right\}^{1 / p} \geq \frac{q_{t}\left(r_{t}^{-q_{t}}-r_{t}^{q_{t}}\right)}{r_{t}^{J}}\left\{\begin{array}{c}
\left(\int_{0}^{2 \pi}\left|\frac{\mu_{J+1, J}}{\left(1-r_{t}^{2 q_{t}} e^{i q_{t} \theta}\right)^{J+1}}\right|^{p} d \theta\right)^{1 / p} \\
-\sum_{h=1}^{J}\left(\int_{0}^{2 \pi}\left|\frac{\mu_{h, J}}{\left(1-r_{t}^{2 q_{t}} e^{i q_{t} \theta}\right)^{h}}\right|^{p} d \theta\right)^{1 / p}
\end{array}\right\} \\
>\frac{q_{t}^{J}\left(r_{t}^{-q_{t}}-r_{t}^{q_{t}}\right)}{r_{t}^{J}\left(1-r_{t}^{2 q_{t}}\right)^{J+1-1 / p}}\left\{C(J, p)-C_{5}(J, p) 2^{1 / 2}\left(1-r_{t}\right)^{(1-\alpha) / 2}\left(\delta\left(r_{t}\right)\right)^{1 / 2}\right\} .
\end{gathered}
$$

As $t$ tends to infinity, the function $\delta\left(r_{t}\right)$ tends to zero, so that the right-hand side of the last inequality is asymptotically equal to $C(J, p) q_{t}^{1 / p}\left(1-r_{t}\right)^{1 / p-J}$. Therefore

$$
\int_{0}^{2 \pi}\left|b_{t}^{(J)}\left(r_{t} e^{i \theta}\right)\right|^{p} d \theta>C_{1}(J, p) q_{t}\left(1-r_{t}\right)^{1-J_{p}} \quad(p>1) .
$$

On the other hand, for $p>1$, Lemma 6 with (37) can be used to get

$$
\begin{aligned}
\int_{0}^{2 \pi}\left|b_{t}^{(J)}\left(r_{t} e^{i \theta}\right)\right|^{p} d \theta & =\int_{0}^{2 \pi}\left|b_{t}^{(J)}\left(r_{t} e^{i \theta}\right)\right|^{p-1}\left|b_{t}^{(J)}\left(r_{t} e^{i \theta}\right)\right| d \theta \\
& \leq \frac{C(J, p)}{\left(1-r_{t}\right)^{J(p-1)}} \int_{0}^{2 \pi}\left|b_{t}^{(J)}\left(r_{t} e^{i \theta}\right)\right| d \theta \\
& <\frac{C_{2}(J, p)}{\left(1-r_{t}\right)^{J(p-1)}} \frac{q_{t}}{\left(1-r_{t}\right)^{J-1}}=C_{2}(J, p) q_{t}\left(1-r_{t}\right)^{1-J p} .
\end{aligned}
$$

Thus, Lemma 9 is proved. 
Lemma 5 applies to Blaschke products $B(z)$ in general. We note the following immediate corollary which is relevant to the particular subproducts of $B(z)$ with the factor $b_{t}(z)$ omitted.

Corollary 1. Let $B(z)$ be a Blaschke products, and let $B_{t}(z)$ denote the subproduct of $B(z)$ with the factor $b_{t}(z)$ omitted, where $b_{t}(z)$ is defined by (26) with $t$ in place of $k$. Then

$$
\int_{0}^{2 \pi}\left|B_{t}^{(J)}\left(r_{t} e^{i \theta}\right)\right|^{p} d \theta<C(J, p) \sum_{\substack{k=1 \\ k \neq t}}^{\infty} \frac{q_{k}\left(1-r_{k}\right)^{p}}{\left(1-r_{k} r_{t}\right)^{(J+1) p-1}}
$$

for $1 /(J+1)<p \leq 1 / J$.

We can now complete the proof of Theorem 3. Write

$$
B(z)=b_{t}(z) \prod_{\substack{k=1 \\ k \neq t}}^{\infty} b_{k}(z)=b_{t}(z) B_{t}(z) \quad(t \in N) .
$$

Then Leibnitz's formula shows that

$$
B^{(n)}(z)=\sum_{m=0}^{n} C_{m}^{n} b_{t}^{(m)}(z) B_{t}^{(n-m)}(z) \quad(n \in \mathbf{N}) .
$$

Since $p \leq 1$, then for $r=r_{t}$, we get

$$
\begin{aligned}
I_{n}\left(r_{t}\right) \geq & \int_{0}^{2 \pi}\left|b_{t}^{(n)}\left(r_{t} e^{i \theta}\right)\right|^{p}\left|B_{t}\left(r_{t} e^{i \theta}\right)\right|^{p} d \theta \\
& -\sum_{m=0}^{n-1} C_{m}^{n} \int_{0}^{2 \pi}\left|b_{t}^{(m)}\left(r_{t} e^{i \theta}\right)\right|^{p}\left|B_{t}^{(n-m)}\left(r_{t} e^{i \theta}\right)\right|^{p} d \theta .
\end{aligned}
$$

Under the conditions (28) and (29) on $r_{k}$ and $q_{k}$, respectively, we can easily (see [3], pp. 93-94) show that $\left|B_{t}\left(r_{t} e^{i \theta}\right)\right|>1 / 2$. Then by using Lemma 9 in the case $J=n$ we obtain

$$
\int_{0}^{2 \pi}\left|b_{t}^{(n)}\left(r_{t} e^{i \theta}\right)\right|^{p}\left|B_{t}\left(r_{t} e^{i \theta}\right)\right|^{p} d \theta>C_{1}(n, p) q_{t}\left(1-r_{t}\right)^{1-n p} .
$$

In the case $m=0$, Corollary 1 yields, for $J=n$, that

$$
\int_{0}^{2 \pi}\left|b_{t}\left(r_{t} e^{i \theta}\right)\right|^{p}\left|B_{t}^{(n)}\left(r_{t} e^{i \theta}\right)\right|^{p} d \theta<C(n, p) \sum_{\substack{k=1 \\ k \neq t}}^{\infty} \frac{q_{k}\left(1-r_{k}\right)^{p}}{\left(1-r_{k} r_{t}\right)^{(n+1) p-1}} .
$$


Also, since $\quad n p / m=n(m+1) p /(m(m+1))>1 /(m+1) \quad$ and $\quad 1 /(n-m) \geq$ $n p /(n-m)=n(n-m+1) p /((n-m)(n-m+1))>1 /(n-m+1)$, then by applying Hölder's inequality in the cases $1 \leq m \leq n-1$, we obtain from Lemma 9 with Corollary 1 that

$$
\begin{aligned}
\sum_{m=0}^{n-1} & C_{m}^{n} \int_{0}^{2 \pi}\left|b_{t}^{(m)}\left(r_{t} e^{i \theta}\right)\right|^{p}\left|B_{t}^{(n-m)}\left(r_{t} e^{i \theta}\right)\right|^{p} d \theta \\
& <\sum_{m=0}^{n-1} C(m, n, p)\left\{q_{t}\left(1-r_{t}\right)^{1-n p}\right\}^{m / n}\left\{\sum_{\substack{k=1 \\
k \neq t}}^{\infty} \frac{q_{k}\left(1-r_{k}\right)^{n p /(n-m)}}{\left(1-r_{k} r_{t}\right)^{n p+n p /(n-m)-1}}\right\}^{(n-m) / n} .
\end{aligned}
$$

Note that,

$$
\frac{q_{k}\left(1-r_{k}\right)^{n p /(n-m)}}{\left(1-r_{k} r_{t}\right)^{n p+n p /(n-m)-1}}<q_{k}\left(1-r_{k}\right)^{1-n p} \quad(k=1,2, \ldots, t-1),
$$

and

$$
\frac{q_{k}\left(1-r_{k}\right)^{n p /(n-m)}}{\left(1-r_{k} r_{t}\right)^{n p+n p /(n-m)-1}}<\frac{q_{k}\left(1-r_{k}\right)^{n p /(n-m)}}{\left(1-r_{t}\right)^{n p+n p /(n-m)-1}} \quad(k=t+1, t+2, \ldots),
$$

since $1-r_{k} r_{t}>1-r_{t}$ and $n p+n p /(n-m)-1>0$.

Therefore, the left-hand side of (43) is bounded above by

$$
\begin{array}{rl}
\sum_{m=0}^{n-1} & C(m, n, p)\left\{q_{t}\left(1-r_{t}\right)^{1-n p}\right\}^{m / n} \\
& \times\left\{\sum_{k=1}^{t-1} q_{k}\left(1-r_{k}\right)^{1-n p}+\sum_{k=t+1}^{\infty} \frac{q_{k}\left(1-r_{k}\right)^{n p /(n-m)}}{\left(1-r_{t}\right)^{n p+n p /(n-m)-1}}\right\}^{(n-m) / n} .
\end{array}
$$

Thus, from (40), (41) and (44) we obtain

$$
\begin{aligned}
I_{n}\left(r_{t}\right)> & q_{t}\left(1-r_{t}\right)^{1-n p} \times\left\{C_{1}(n, p)-\sum_{m=0}^{n-1} C(m, n, p)\right. \\
& \left.\times\left\{\sum_{k=1}^{t-1} \frac{q_{k}\left(1-r_{k}\right)^{1-n p}}{q_{t}\left(1-r_{t}\right)^{1-n p}}+\sum_{k=t+1}^{\infty} \frac{q_{k}\left(1-r_{k}\right)^{n p /(n-m)}}{q_{t}\left(1-r_{t}\right)^{n p /(n-m)}}\right\}^{(n-m) / n}\right\} .
\end{aligned}
$$

For any $\Omega>0$ (however small) there is a corresponding number $K=K(\Omega)$ (with $K>0)$ such that

$$
\frac{\delta\left(r_{k}\right)}{\delta\left(r_{t}\right)}<(1+\Omega)^{t-k} \quad(t>k>K) .
$$


For large values of $t$ and $K$, we have

$$
\sum_{k=1}^{t-1} \frac{q_{k}\left(1-r_{k}\right)^{1-n p}}{q_{t}\left(1-r_{t}\right)^{1-n p}}<\frac{4(1+\Omega) \sigma^{n p+\alpha-1}}{1-(1+\Omega) \sigma^{n p+\alpha-1}},
$$

for a suitable choice of $\sigma$ in $(0,1)$ so that $(1+\Omega) \sigma^{n p+\alpha-1}<1$.

Further, for $0 \leq m \leq n-1(n \in \boldsymbol{N})$, we get

$$
\sum_{k=t+1}^{\infty} \frac{q_{k}\left(1-r_{k}\right)^{n p /(n-m)}}{q_{t}\left(1-r_{t}\right)^{n p /(n-m)}}<\frac{2 \sigma^{(n p /(n-m)-\alpha)}}{1-\sigma^{(n p /(n-m)-\alpha)}} .
$$

Hence, for a suitable choice of $\sigma$ in $(0,1)$ so that

$$
\sum_{m=0}^{n-1} C(m, n, p)\left\{\frac{4(1+\Omega) \sigma^{n p+\alpha-1}}{1-(1+\Omega) \sigma^{n p+\alpha-1}}+\frac{2 \sigma^{(n p /(n-m)-\alpha)}}{1-\sigma^{(n p /(n-m)-\alpha)}}\right\}^{(n-m) / n} \leq \frac{C_{1}(n, p)}{2},
$$

we obtain from (45), (46) and (47) that

$$
I_{n}\left(r_{t}\right)>\frac{C_{1}(n, p)}{2} q_{t}\left(1-r_{t}\right)^{1-n p}>\frac{C_{1}(n, p)}{4} \frac{\delta\left(r_{t}\right)}{\left(1-r_{t}\right)^{n p+\alpha-1}} \geq \frac{\varepsilon\left(r_{t}\right)}{\left(1-r_{t}\right)^{n p+\alpha-1}},
$$

if $C_{1}(n, p) \geq 4$. If not, then by initially replacing $\varepsilon(r)$ by $\left(4 /\left(C_{1}(n, p)\right)\right) \varepsilon(r)$ we get

$$
I_{n}\left(r_{t}\right)>\frac{\varepsilon\left(r_{t}\right)}{\left(1-r_{t}\right)^{n p+\alpha-1}}=\frac{\varepsilon(r)}{(1-r)^{n p+\alpha-1}} \quad\left(r=r_{t}\right) .
$$

This completes the proof of Theorem 3 .

\section{General counterexamples in cases when $p>1 / n$}

The next theorem shows that the inequality (24) with the condition (3) is the best obtainable for general infinite Blaschke products.

THEOREM 4. For a natural number $n$ let $\alpha \in(0,1 /(n+1))$, and let $\varepsilon(r)$ be a given positive function which is continuous on $(0,1)$ and satisfies the condition (3). Then we can find an infinite Blaschke product $B(z)$ whose zeros $\left\{a_{k}\right\}$ satisfy the associated convergence condition (2), and for any number $p>1 / n$ we have

$$
I_{n}(r)=\int_{0}^{2 \pi}\left|B^{(n)}\left(r e^{i \theta}\right)\right|^{p} d \theta>\frac{\varepsilon(r)}{(1-r)^{n p+\alpha-1}},
$$

for a sequence of values $r$ increasing to 1 . 
Proof. In order to prove this theorem we proceed by defining $B(z)$ as in (26), where the conditions (27) and (28) hold.

As previously, we shall prove our result in terms of $\delta$ rather than $\varepsilon$, which satisfies the conditions (i), (ii) and (iii) which have been mentioned in Lemma 7. Further, we may assume, without loss of generality, that the definition (29) with the condition (30) hold.

Write $B(z)$ as in (39). Then Leibnitz's formula can be used together with Lemma 6 to obtain

$$
\left|\sum_{m=0}^{n-1} C_{m}^{n} b_{t}^{(m)}\left(r_{t} e^{i \theta}\right) B_{t}^{(n-m)}\left(r_{t} e^{i \theta}\right)\right|<\frac{C_{3}(n)}{\left(1-r_{t}\right)^{n}} \quad\left(0<r_{t}<1\right) .
$$

Thus with $p>1 / n$ we can choose a number $\gamma$ such that $1 /(n+1)<\gamma \leq 1 / n$, then (43) can be used with $p$ replaced by $\gamma$ to get

$$
\begin{aligned}
& \int_{0}^{2 \pi}\left|\sum_{m=0}^{n-1} C_{m}^{n} b_{t}^{(m)}\left(r_{t} e^{i \theta}\right) B_{t}^{(n-m)}\left(r_{t} e^{i \theta}\right)\right|^{p} d \theta \\
& \quad<\sum_{m=0}^{n-1} C(m, n, \gamma, p)\left\{\frac{\left(q_{t}\left(1-r_{t}\right)^{1-n \gamma}\right)^{m / n}}{\left(1-r_{t}\right)^{n(p-\gamma)}}\left(\sum_{\substack{k=1 \\
k \neq t}}^{\infty} \frac{q_{k}\left(1-r_{k}\right)^{n \gamma /(n-m)}}{\left(1-r_{k} r_{t}\right)^{n \gamma+n \gamma /(n-m)-1}}\right)^{(n-m) / n}\right\} .
\end{aligned}
$$

In those cases where $1 / n<p \leq 1$ we can use (41) and (51) to show that

$$
\begin{aligned}
I_{n}\left(r_{t}\right)= & \int_{0}^{2 \pi}\left|B^{(n)}\left(r_{t} e^{i \theta}\right)\right|^{p} d \theta \\
\geq & \int_{0}^{2 \pi}\left|b_{t}^{(n)}\left(r_{t} e^{i \theta}\right)\right|^{p}\left|B_{t}\left(r_{t} e^{i \theta}\right)\right|^{p} d \theta-\int_{0}^{2 \pi}\left|\sum_{m=0}^{n-1} C_{m}^{n} b_{t}^{(m)}\left(r_{t} e^{i \theta}\right) B_{t}^{(n-m)}\left(r_{t} e^{i \theta}\right)\right|^{p} d \theta \\
> & q_{t}\left(1-r_{t}\right)^{1-n p} \times\left\{C_{1}(n, p)-\sum_{m=0}^{n-1} C(m, n, \gamma, p)\right. \\
& \left.\times\left\{\sum_{k=1}^{t-1} \frac{q_{k}\left(1-r_{k}\right)^{1-n \gamma}}{q_{t}\left(1-r_{t}\right)^{1-n \gamma}}+\sum_{k=t+1}^{\infty} \frac{q_{k}\left(1-r_{k}\right)^{n \gamma /(n-m)}}{q_{t}\left(1-r_{t}\right)^{n \gamma /(n-m)}}\right\}^{(n-m) / n}\right\}
\end{aligned}
$$

which is similar to (45) with $p$ replaced by $\gamma$ inside the brackets \{\} . Therefore, the same technique that was used in Theorem 3 to establish the result (48) can be applied again to get

$$
I_{n}\left(r_{t}\right)>\frac{\varepsilon\left(r_{t}\right)}{\left(1-r_{t}\right)^{n p+\alpha-1}},
$$

where $\lim _{t \rightarrow \infty} \varepsilon\left(r_{t}\right)=0$ and $1 / n<p \leq 1$. 
On the other hand, when $p>1$, the technique that was used in Theorem 3 can be used, once again, together with Hölder's inequality with (41) and (51) to show that

$$
\begin{aligned}
& \left\{I_{n}\left(r_{t}\right)\right\}^{1 / p}>\left\{q_{t}\left(1-r_{t}\right)^{1-n p}\right\}^{1 / p} \\
& \quad \times\left\{\left(C_{1}(n, p)\right)^{1 / p}-\sum_{m=0}^{n-1}(C(m, n, \gamma, p))^{1 / p}\left\{\begin{array}{l}
\sum_{k=1}^{t-1} \frac{q_{k}\left(1-r_{k}\right)^{1-n \gamma}}{q_{t}\left(1-r_{t}\right)^{1-n \gamma}} \\
+\sum_{k=t+1}^{\infty} \frac{q_{k}\left(1-r_{k}\right)^{n \gamma /(n-m)}}{q_{t}\left(1-r_{t}\right)^{n \gamma /(n-m)}}
\end{array}\right\}\right. \\
& >\left\{\frac{\varepsilon\left(r_{t}\right)}{\left(1-r_{t}\right)^{n p+\alpha-1}}\right\}^{1 / p} .
\end{aligned}
$$

Therefore

$$
I_{n}\left(r_{t}\right)>\frac{\varepsilon\left(r_{t}\right)}{\left(1-r_{t}\right)^{n p+\alpha-1}}=\frac{\varepsilon(r)}{(1-r)^{n p+\alpha-1}},
$$

where $r=r_{t}, \lim _{t \rightarrow \infty} \varepsilon\left(r_{t}\right)=0$ and $p>1$.

This completes the proof of Theorem 4 .

\section{Integral mean inequalities valid everywhere on $\left[r_{0}, 1\right)$}

We note that, if $\varepsilon(r)$ is fairly small, then Theorems 3 and 4 can hold everywhere on an interval $\left[r_{0}, 1\right)$. As a sample of what is possible, we obtain the following theorem.

THEOREM 5. For a natural number $n$, let $\alpha \in(0,1 /(n+1))$, and let $\varepsilon(r)$ be a given continuous function on $(0,1)$ and which for a constant number $\eta(>1)$, satisfies

$$
0<\varepsilon(r) \leq\left\{\log (1-r)^{-1}\right\}^{-\eta} \quad(0<r<1),
$$

then, for some $r_{0}$ in $(0,1)$, and for any number $p>(1-\alpha) / n$, we can find an infinite Blaschke product $B(z)$ whose zeros satisfy the associated convergence condition (2) such that

$$
I_{n}(r)=\int_{0}^{2 \pi}\left|B^{(n)}\left(r e^{i \theta}\right)\right|^{p} d \theta>\frac{\varepsilon(r)}{(1-r)^{n p+\alpha-1}}, \quad\left(r_{0} \leq r<1\right) .
$$

Proof. Since $\varepsilon(r)$ is assumed to satisfy the condition (54), then we can follow through the proofs of Theorems 3 and 4 by putting

$$
\delta(r)=\left\{\log (1-r)^{-1}\right\}^{-\eta} .
$$


So, for the suitably chosen constant $\sigma$ in $(0,1)$ we have

$$
\sum_{k=1}^{\infty} \delta\left(r_{k}\right)=\sum_{k=1}^{\infty}\left\{(k-1) \log \left(\frac{1}{\sigma}\right)+\log \left(\frac{1}{1-r_{1}}\right)\right\}^{-\eta}<\infty,
$$

as long as we assume that $\eta>1$ and

$$
1-r_{k+1}=\sigma\left(1-r_{k}\right) \quad(k=1,2, \ldots) .
$$

Consequently, there is no need to take a subsequence $\left\{r_{k_{s}}\right\}$ of $\left\{r_{k}\right\}$ to ensure the convergence of the series $\sum_{s=1}^{\infty} \delta\left(r_{k_{s}}\right)$.

We point out that $B(z)$ was considered to have $q_{k}$ zeros, for which we obtain

$$
\sum_{k=1}^{\infty}\left(1-\left|a_{k}\right|\right)^{\alpha}=\sum_{k=1}^{\infty} q_{k}\left(1-r_{k}\right)^{\alpha}<\infty \quad\left(0<\alpha<\frac{1}{n+1}\right)
$$

where $q_{k}=\left[\left(1-r_{k}\right)^{-\alpha} \delta\left(r_{k}\right)\right]$ for $k=1,2, \ldots$

Thus, we have a Blaschke product $B(z)$ with zeros satisfying the convergence condition (59), while the inequality (55) holds for the sequence of values $r=r_{t}$ increasing to 1 , when $t$ is sufficiently large. However, if for any natural number $t$, we have $r_{t} \leq r<r_{t+1}$, then we can use the increasing property of $I_{n}(r)$ (as a function of $r$ ) to get

$$
I_{n}(r)=\int_{0}^{2 \pi}\left|B^{(n)}\left(r e^{i \theta}\right)\right|^{p} d \theta \geq \int_{0}^{2 \pi}\left|B^{(n)}\left(r_{t} e^{i \theta}\right)\right|^{p} d \theta>\frac{\delta\left(r_{t}\right)}{\left(1-r_{t}\right)^{n p+\alpha-1}}>\frac{\sigma^{n p+\alpha-1} \delta(r)}{(1-r)^{n p+\alpha-1}} .
$$

By redesignating the function $\delta$ or initially writing $\delta(r)=\sigma^{1-n p-\alpha}$. $\left\{\log (1-r)^{-1}\right\}^{-\eta}$ in place of $(56)$, we end the proof of the Theorem.

Thus, we can see that this type of results may be extended by the introducing of a logarithmic scale which ensures the convergence property (57). For example, the condition (54) might be replaced by

$$
0<\varepsilon(r) \leq\left\{\log (1-r)^{-1}\right\}^{-1}\left\{\log \log (1-r)^{-1}\right\}^{-\eta}
$$

for $\eta>1$ and $0<r<1$.

The author would like to express his great appreciation to Dr. C. N. Linden, for his help and continuous encouragement.

\section{REFERENCES}

[1] T. M. Apostol, Mathematical Analysis, Second Edition, Addison-Weslay Publishing, Reading, 1974.

[2] H. O. KIM, Derivatives of Blaschke products, Pacific J. Math., 114 (1984), 175-190.

[ 3 ] M. A. KutBI, Integral means for the first derivative of Blaschke products, Kodai Math. J., 24 (2001), 86-97. 
[ 4 ] C. N. Linden, $H^{p}$-derivatives of Blaschke products, Michigan Math. J., 23 (1976), 43-51.

[5] D. Protas, Blaschke products with derivative in $H^{p}$ and $B^{p}$, Michigan Math. J., 20 (1973), 393-396.

[6] M. Tsuj,, Potential Theory in Modern Function Theory, Chelsea Publishing, New York, 1959.

Department of Mathematics

King Abdulaziz University

P.O. Box 80203

JEDDAH 21589

Kingdom of Saudi Arabia

e-mail: mkutbi@yahoo.com 